\title{
Approach to the Immunosuppressed Patient with Pulmonary Infiltrates
}

\author{
JOSEPH P. LYNCH, III, M.D.
}

Pulmonary complications represent a major cause of morbidity and mortality in immunosuppressed patients (IP). The varied etiologies of pulmonary disease in IPs include pyogenic, mycobacterial and opportunistic infections, drug-induced pulmonary interstitial fibrosis, pulmonary parenchymal involvement by the underlying disease process, and intrapulmonary hemorrhage. With the increasing use of corticosteroid and cytotoxic therapy in a variety of nonmalignant diseases, such as vasculitis, dermatomyositis, connective tissue disorders, pemphigus vulgaris, chronic active hepatitis, and others, recognition of the potential complications associated with their use is imperative.

Unusual and fulminant infections may complicate immunosuppressive therapy. Corticosteroid drugs impair host defenses by several mechanisms, including lymphocyte depletion, defective cellular immunity, inhibition of macrophage migration and phagocytosis, and polymorphonuclear leukocyte dysfunction. ${ }^{1}$ Prolonged corticosteroid therapy in animals may predispose to severe fungal and protozoan infections. ${ }^{2,3}$ Alkylating agents and azothiaprine similarly $\mathrm{f}$.edispose to infectious complications, primarily by their effects on reducing granulocyte number. ${ }^{4}$

The development of pulmonary infiltrates in IP requires urgent and aggressive evaluation, since infections may rapidly overwhelm a host with impaired defenses. Delay in therapy by only a few days may preclude a successful outcome. However, the empiric use of multiple antimicrobial and antimycotic agents to cover all possible infectious etiologies may be hazardous due to the potential toxic effects of these drugs. The serious toxicity of antifungal therapy with amphotericin B makes empiric therapy in undiagnosed infections inadvisable,

Address for reprints: Joseph B. Lynch, III, M.D., the Pulmonary Division, Department of Internal Medicine, University of Michigan Medical Center, Ann Arbor, Ml 48109.
From the Pulmonary Division. Department of Internal Medicine, University of Michigan Medical Center. Ann Arbor, Michigan

although its value in documented fungal infections is unquestioned. This review summarizes the major infectious and noninfectious pulmonary complications seen in IPs, with emphasis on clinical manifestations, differential diagnosis, diagnostic evaluation, and management.

\section{Bacterial Pneumonia}

Pneumonia represents the major cause of morbidity and mortality in immunocompromised host. ${ }^{\mathbf{5} .6}$ Although the incidence of fungal and opportunistic infections are higher in this population, bacteria remain the most common causative agents. ${ }^{6.7}$ Several features differentiate bacterial pneumonia in IPs from normal hosts: the higher mortality, ${ }^{8}$ greater incidence of gram-negative organisms, ${ }^{6.7}$ and relative dearth of physical findings of pneumonia in IPs. ${ }^{6,9}$ The predominance of gramnegative organisms in patients with neoplasia is affirmed by demonstrating 217 gram-negative pneumonias out of a total of 243 bacillary pneumonias seen in one cancer hospital over a six-year period. ${ }^{9}$ Although fever was present in 195 of 217 gram-negative pneumonias, cough and sputum production were present in a minority. ${ }^{9}$ Further, physical findings were scanty, and findings of consolidation were present in only $18 \%$. Roentgenograms rarely exhibited the classical lobar pattern typical of normal hosts with bacterial suppurative infection, and frequently involved multiple lobes bilaterally. Diagnosis was further impeded by the scarcity of roentgenographic findings in neutropenic patients, presumably related to their inability to mount an inflammatory response. The high incidence of bacteremia (32\%) in that series em- 
phasizes the importance of obtaining blood cultures in all IPs with pneumonia.

Others have affirmed the frequent lack of classic signs and symptoms in IPs. ${ }^{6}$ Although fever was present in $98 \%$ of 52 cases of pneumonia in acute leukemia patients, cough and sputum production were noted in less than two-thirds, and consolidation, in a minority. Sputum was produced in only 30 of 52 episodes of pneumonia, and polymorphonuclear leukocytes were usually scarce. However, the gram stain was quite accurate in suggesting the causative organism(s).

Although the prognosis is generally better with grampositive pneumonias, ${ }^{7}$ the course in some IP can be fulminant. Reduction in corticosteroid or immunosuppressive therapy may improve prognosis in renal transplant patients with severe bacterial pneumonia. ${ }^{10}$ Due to the high mortality and morbidity from bacillary pneumonias in IP, early coverage with antibiotics, reduction in degree of immunosuppression, and granulocyte transfusions, when required, are indicated. Broad spectrum parenteral therapy to include an aminoglycoside, as well as aerobic and anaerobic coverage is indicated at the onset of symptoms. Delaying therapy pending a definitive cultural diagnosis is to be strongly discouraged.

\section{Legionnaire's}

Pulmonary infection with Legionella pneumophilia may be more common in IPs, and tends to run a more fulminant course. ${ }^{11}$ In 8 IPs with pneumonia caused by $L$. pheumophilia, the initial presentation was indistinguishable from other bacterial pneumonias. ${ }^{11}$ All had fever and malaise, and all but one manifested chills. Although most produced sputum, in only a single case were sputum leukocytes increased. In all cases, sputum cultures grew normal flora. Chest roentgenograms initially showed patchy infiltrates limited to one lobe, with rapid progression over the next few days to consolidation and bilateral disease in the majority. Abscess formation occurred in two, and four developed respiratory failure. Diagnosis was made in four cases by open lung biopsy, by either cultures or immunofluorescent antibody (FA) testing, and in the others by cultures of bronchial washings, direct FA staining of sputum and bronchial washings, and concurrent serologic titer rises. Unlike normal hosts, response to oral erythromycin was poor, but parenteral erythromycin was associated with a favorable outcome. Further, in two patients responding slowly to erythromycin, the addition of rifampin accelerated the resolution. Hence, for IP with pneumonia, early diagnosis via invasive procedures or FA testing for Legionella should be considered. Empiric therapy with parenteral erythromycin $500 \mathrm{mg}$ every six hours is indicated in such patients while awaiting biopsy, or in whom no biopsy is planned. In those with a definitive diagnosis showing a slow response to erythromycin, rifampin should be added for its possible synergistic effect.

\section{Pittsburgh Agent}

The recent report of an acute purulent pneumonia in two renal transplant recipients due to the newly described "Pittsburgh Agent," 12 emphasizes early recognition of hitherto unsuspected organisms causing infection in IPs. Since standard cultural techniques may fail to demonstrate the organism, specific FA stains of alveolar exudate or lung tissue should be performed to confirm the diagnosis. Although optimal therapy is not clear, beta-lactam antibiotics, such as penicillin, cephalothin, and cefoxitin appear to be effective in vitro. ${ }^{12}$

\section{Nocardiosis}

Pulmonary infection with the gram-positive, partially acid-fast coccobacilli of the Nocardia species can be exceedingly difficult to diagnose. The organisms can be difficult to visualize when gram stained, and are notoriously difficult to culture. ${ }^{5}$ Hence, sputum cultures are usually negative. Positive blood cultures are exceedingly rare, even with disseminated disease. ${ }^{5}$ Roentgenographic findings are variable, including lobar or segmental consolidation, extensive broncopneumonia with multiple abscesses, focal nodules, cavities, or a miliary pattern. ${ }^{5}$ Histopathology is variable, and can show extensive suppurative disease with polymorphonuclear leukocyte infiltration and abscess formation, or a chronic granulomatous reaction with multinucleated giant cells and mononuclear cells. ${ }^{5}$ Due to the low yield of noninvasive techniques, open lung biopsy is frequently required for definitive diagnosis. Sulfonamide therapy is highly effective, but must be continued for 6 to 12 months to preclude relapses. ${ }^{5}$

\section{Fungal Infections}

Fungal pneumonia in IPs can be highly lethal, with a rapid course, tendency to disseminate, and extreme difficulty in making a definitive diagnosis antemortem. Several factors appear to predispose to invasive mycotic infection, including prolonged leukopenia, corticosteroid or cytotoxic drug therapy, and prolonged antibiotic therapy. ${ }^{13,14}$

\section{Aspergillus Species}

Invasive aspergillosis is particularly difficult to diagnose due to extreme difficulty in identifying or culturing the fungus antemortem. In one autopsy series of leukemics, Aspergillus represented the most common fungus identified during autopsy, but in none of the 10 cases were antemortem cultures positive, despite extensive pneumonia or even dissemination. ${ }^{14}$ Others have confirmed the unreliability of antemortem cultures in 
documented aspergillosis. ${ }^{15}$ In 93 cases of Aspergillus infection complicating neoplasia, cultures were positive in only seven of 59 antemortem cultures of sputum, throat, or tracheal secretions. ${ }^{15}$ In no cases were blood, urine, or cerebrospinal fluid (CSF) cultures positive, even with dissemination of extensive cerebral infection. Hence, demonstration of the characteristic acute branching septate hyphae on hematoxylin-eosin or methenamine-silver stained tissue sections of biopsy material is generally required for diagnosis.

Aspergillus is a classic invader of the lung. Pulmonary involvement was documented in 90 of 93 cases of invasive Aspergillus infections reported by Meyer et al. ${ }^{15}$ The most common clinical pattern for aspergillus infection was unremitting fever and persistent or increasing pulmonary infiltrates despite antibiotic therapy. The most common types of lung involvement are necrotizing bronchopneumonia and hemorrhagic infarction ${ }^{15} \mathrm{sec}$ ondary to the strong propensity of Aspergillus to invade vasculature, with the development of in situ thromboses and multiple infarcts. Aspergillus also commonly invades the central nervous system (CNS) with abscess formation.

In the presence of pulmonary infiltrates or CNS findings, the isolation of Aspergillus species from any cultural site should strongly suggest infection in IPs. Repeated isolation or demonstration of aspergillus in histologic sections mandates amphotericin B therapy. However, aspergillus can be a contaminant, and a single isolation in the absence of clinical disease does not warrant therapy.

\section{Phycomycetes}

Phycomycetes resemble aspergillus by their propensity to involve the lung and CNS, their predilection to invade vasculature with intraluminal thromboses, and the clinical hallmarks of hemoptysis and pulmonary infarction. Phycomycetes is notoriously difficult to culture antemortem, even in tissue specimens. Of 21 patients with pulmonary phycomycosis reported by Meyer, all eight with antemortem cultures of respiratory secretions were negative, and all 13 postmortem lung cultures were negative, despite evidence of pulmonary phycomycosis. ${ }^{16}$ Hence, cultures are unreliable even with disseminated or extensive pulmonary phycomycosis, and invasive procedures to procure tissue for demonstration of the classic, large, nonspecific hyphae are required in most cases. Although mortality with invasive phycomycosis is high, early therapy with amphotericin B and/or surgical resection has been successful. ${ }^{17}$

\section{Candida Species}

Infections with Candida species are among the most common invasive mycoses in IPs. Predisposing factors to the development of invasive candidiasis include profound granulocytopenia, prolonged antibiotic therapy, corticosteroids, the presence of gastrointestinal mucosal lesions, and intravenous catheters. ${ }^{5}$ Pulmonary involvement is frequent, and may occur as candida infection limited to the lung, or in the setting of disseminated candidiasis. Unfortunately, the roentgenographic pattern of candidial pneumonia is not specific, and may show patchy bronchopneumonia, lobar consolidation, nodules, cavities, or diffuse miliary mottling. ${ }^{5}$ Further, none of the serologic tests are reliable in either diagnosing or excluding candidiasis. ${ }^{5}$ Diagnosis of invasive candidiasis by cultures of respiratory secretions is extremely unreliable, particularly in patients with prior antibiotic therapy, because of the high colonization rate without representing deep tissue invasion. ${ }^{13}$ Although the presence of pseudohyphae on sputum is suggestive of deep rather than superficial infection, this finding is not wholly reliable. Hence, the most accurate way of diagnosing candidal pneumonia is the demonstration of fungi invading pulmonary parenchyma on biopsy specimens. ${ }^{5}$ In cases where pulmonary parenchyma is not obtained, a high probability of candidal pneumonia can be inferred in the presence of signs of dissemination, such as multiple positive blood cultures or cultures from multiple sites. Culturing candida from metastatic skin abscesses, multiple blood cultures, or the finding of typical fluffy exudates on retinal exam makes dissemination likely, and warrants treatment with amphotericin B.

\section{Histoplasma capsulatum}

The spectrum of histoplasmosis ranges from benign asymptomatic pneumonitis to fatal dissemination. Although data are limited in patients with underlying immune deficiency states, it seems clear that the risk of fatal and/or disseminated histoplasmosis is higher in this population. ${ }^{18-20}$ Davies et al reported disseminated histoplasmosis in eight IPs, and noted the onset of spiking fevers within three months of the initiation of corticosteroid therapy in five. ${ }^{18}$ The clinical picture was nonspecific, but all had spiking fevers and no patient produced sputum. None had any history or signs of acute respiratory illness preceding dissemination. Although in four cases the chest roentgenogram was normal at the onset of fever, all subsequently developed abnormalities. The most common roentgenographic appearance was diffuse interstitial infiltrates, but diffuse nodules were also seen. Serologies were diagnostic in only one of four tested. The relatively high yield of cultures of tissue and blood, in contradistinction to Aspergillus and Phycomycetes, however, is worth emphasizing. Blood cultures were positive for $H$. capsulatum in 4 of 7 patients, and bone marrow cultures were positive in 3 of 4 patients. Induced sputum and urine yielded pos- 
itive results in 3 of 6 and 1 of 8 cases, respectively. The high yield of cultured specimens is confirmed by a recent report of 22 consecutive patients with disseminated histoplasmosis undergoing trephine bone marrow biopsy with cultures. ${ }^{19}$ Bone marrow biopsy demonstrated typical fungi in tissue in 15 patients, and cultures were positive despite negative histology in an additional four. Two additional biopsies showed granuloma suggestive of fungal infection. The importance of performing methenamine-silver stains in addition to hematoxylin-eosin was demonstrated by finding that in 7 patients with negative hematoxylin-eosin stains, silver stains demonstrated histoplasmosis. ${ }^{19}$

Oropharyngeal ulcers and skin lesions are common in disseminated histoplasmosis and should be sought and biopsied, if present. ${ }^{20}$

\section{Cryptococcus}

Cryptococcal infection is strikingly increased in IPS, and dissemination is common. Pulmonary involvement is seen in virtually all cases, and is generally manifested as diffuse interstitial pneumonitis on roentgenogram. The vast majority are febrile, and neurologic symptoms, although sometimes subtle, may be present. ${ }^{21}$ Cultures of blood, urine, and sputum are positive for $10-30 \%$ of all cases, but the highest yield may be from CSF. ${ }^{21}$ In 2 of 46 cases reported by Kaplan et al, initial diagnosis was made by urine cultures. ${ }^{21}$ Histology may be variable, and can show histiocytic-lymphocytic infiltration, granulomas, or frank suppurative pneumonia with prominent neutrophilic response and abscess formation. ${ }^{21}$ Hence, careful cultural techniques and staining for fungi with methenamine-silver should be performed in any biopsy material obtained, regardless of histologic appearance.

The availability of a latex-agglutination slide test for cryptococcal antigen in sera may provide a rapid diagnosis, with a sensitivity rate in excess of $90 \% .{ }^{22}$ However, falsenegative tests can occur in the face of extensive infection, and should not preclude invasive procedures to obtain tissue in patients with a compatible clinical diagnosis. Because of its strong propensity to invade the CNS, lumbar puncture to include India ink staining for Cryptococcus and cryptococcal antigen determination should be performed in any IP with interstitial pneumonitis with impaired mental status, neurologic find ings, or headache.

\section{Blastomyces dermatitidis}

Pulmonary blastomycosis in IPS can be a diagnostic dilemma. Sputum cultures are frequently negative, and serologies and skin tests are wholly unreliable. ${ }^{23}$ Roentgenographic findings are nonspecific, and can in- clude diffuse reticulonodular infiltrates, patchy bronchopneumonia, or lobar consolidation. Skin lesions frequently accompany Blastomyces pulmonary infections and should be cultured and biopsied, if present. The propensity to invade the prostate gland makes prostatic massage for culturing secretions and urine culture warranted in suspected cases. Papanicolau's stain test of sputum or bronchial washings may reveal the characteristic Blastomyces organism. ${ }^{23}$ When the above studies are nondiagnostic, invasive techniques to obtain lung parenchyma may be necessary.

\section{Mycobacterial Infection}

Mycobacterial infections are more common and can be fulminant in IPs. In 201 tuberculous infections in patients with neoplasia, Kaplan noted a higher prevalence rate, strikingly high mortality, and greater incidence of dissemination in patients with lymphoreticular malignancies. ${ }^{24}$ Feld et al reported 59 cases of mycobacterial disease complicating pregnancy, including 30 cases of atypical mycobacteria. ${ }^{25}$ In contrast to Kaplan's series, dissemination only occurred in three cases.

Most large series of pulmonary infiltrates in IPs report only occasional mycobacterial infections, however. In two series of open lung biopsies in IPs, tuberculous infections were found in 1 of $78^{26}$ and 2 of $70^{27}$ biopsies. Similarly, data from transbronchoscopic biopsy procedures confirm only two tuberculous infections in 190 patients. ${ }^{8.28-31}$

In summary, although mycobacterial infection complicating immunosuppression is uncommon, when it occurs, it can be lethal. Since antituberculous therapy is of low toxicity, empiric therapy in IPS with pulmonary infiltrates can be considered while awaiting a definitive diagnosis. However, biopsy procedures should be performed, when possible, in IPs with pulmonary infiltrates of unknown etiology, due to the strong likelihood of other opportunistic or fungal agents.

\section{Pneumocystis carinii}

$P$. carinii is a common cause of diffuse bilateral infiltrates in IPs. ${ }^{32-34}$ Typically, patients have fever, nonproductive cough, progressive dyspnea, hypoxemia, and scanty auscultatory findings. ${ }^{35}$ Chest roentgenograms classically show patchy reticulonodular infiltrates progressing to diffuse alveolar consolidation and air bronchograms. ${ }^{36}$ However, atypical features with unilateral predominance, lobar or segmental consolidation, or pseudonodular patterns, can rarely be seen. ${ }^{36}$ Pleural effusions and lymphadenopathy are distinctly uncommon, and cavitation is never seen. ${ }^{36}$ The clinical course varies from an inapparent pneumonitis to fulminant respiratory failure leading to death. Serologic testing is of no value, 
either in confirming or excluding the diagnosis. ${ }^{32,37}$ Since the organism has never been cultured, diagnosis requires visualization of the typical cysts on methenamine-silver stains of bronchial secretions or biopsy material. The yield on sputum or tracheal aspirates is extraordinarily low, ${ }^{33,35}$ but bronchial brushings reveal the organism in a majority of cases. " Open lung biopsy has a higher yield ${ }^{32,35}$ Typical histologic features include alveolar septal thickening, hyaline membranes, and a foamy proteinaceous exudate filling alveolar spaces. ${ }^{5,33}$ Treatment for $P$. carinii is effective if initiated early, and survival is directly related to duration of therapy with either pentamadine or a combination of trimethaprim-sulfamethoxazole (TMP-SMZ). ${ }^{38}$ Since TMP-SMZ has little morbidity, its empiric initiation in suspected cases of pneumocystosis seems warranted.

\section{Viral Pneumonitis}

Cytomegalovirus (CMV), herpes simplex virus (HSV) and varicella can cause pneumonia in IPs. The clinical course of pneumonia due to these viruses is highly variable, ranging from asymptomatic to fulminant respiratory insufficiency leading to death. The chest roentgenogram is nonspecific and may show patchy bronchopneumonia, diffuse interstitial pneumonitis, or alveolar consolidation. ${ }^{5}$ Tachypnea, nonproductive cough, dyspnea, and low-grade fever are classic, but not uniformly present. Although skin lesions may be present in pneumonia due to CMV and HSV, varicella pneumonia invariably occurs in conjunction with typical skin lesions. ${ }^{18}$ Serologic tests are nonspecific, and titer rises do not correlate with extent of pulmonary involvement. ${ }^{50} 40$ Intranuclear inclusions visualized on Papaniculau's stain test of sputum may suggest the diagnosis, but due to the high incidence of coexistent infections with $P$. carinii, bacterial, and fungal pathogens, invasive techniques to obtain pulmonary parenchyma may be required. The definitive diagnosis of viral pneumonia requires demonstration of characteristic intranuclear inclusions within alveolar cells and/or concomitant viral isolation by culture. ${ }^{5}$ Therapy with adenine arabinoside may be of benefit in extensive HSV infection. ${ }^{5}$ Although no effective treatment is available for CMV and varicella pneumonia, reducing the degree of immunosuppression is recommended. ${ }^{5}$

As in the normal host, viral pneumonias due to influenza, parainfluenza, and adenovirus, can on occasion be fulminant, with development of respiratory distress, disseminated intravascular coagulation, and bronchiolitis obliterans. ${ }^{41}$ Roentgenograms may show diffuse interstitial pneumonia, extensive consolidation, or both. Striking changes of alveolar septal edema, inflammation, and interstitial fibrosis can occur. ${ }^{41}$ Treatment is supportive.

\section{Pulmonary Toxicity}

Pulmonary infiltrates, associated with dyspnea and occasionally frank respiratory failure, may complicate radiation therapy to the thorax..$^{42}$ Chest roentgenograms may show diffuse interstitial and alveolar infiltrates, characteristically confined to the radiation portals and not to anatomical boundaries. ${ }^{42}$ Histologic features include atypical and hyperplastic alveolar lining cells, hyaline membranes, septal thickening and infiltration with mononuclear cells, and fibrosis. ${ }^{42}$ These histopathologic changes, although characteristic of radiation injury, are not specific and can be seen with a wide range of etiologies, including $P$. carinii, viral, and druginduced pneumonitis.

A variety of cytotoxic agents, including busulfan, bleomycin, methotrexate, cyclophosphamide, 6-mercaptopurine, azothiaprine, melphelan, BCNU, and CCNU, can cause acute pneumonitis and chronic interstitial fibrosis, occasionally progressing to fatal respiratory insufficiency. ${ }^{43}$ Pulmonary disease induced by these agents is clinically and roentgenographically indistinguishable from interstitial pneumonitis of infectious and other etiologies.

Busulfan can induce striking pulmonary toxicity, with cough, dyspnea, and roentgenographic changes of chronic interstitial pneumonitis with superimposed alveolar consolidation. ${ }^{44}$ Busulfan is rather unique in its strong propensity to induce striking cytologic dysplasia in bronchial and alveolar epithelium, with the development of atypical bizarre cells with hyperchromatic nuclei. ${ }^{44}$ Finding these characteristic cells on sputum cytology may suggest the diagnosis. The development of busulfan-induced lung injury necessitates withdrawal of the drug.

A syndrome of cough, exertional dyspnea, and infiltrates on chest roentgenograms may be seen within 12 days to five years after initiation of methotrexate therapy. ${ }^{43}$ Chest roentgenographic abnormalities may include linear and reticulonodular infiltrates, widespread alveolar filling, or diffuse nodules. ${ }^{43}$ Histology demonstrates atypical alveolar lining cells, hyaline membranes, interstitial infiltration, and occasional desquamation of cells into alveolar spaces. ${ }^{43}$ Although severe interstitial fibrosis can occur, resolution with discontinuance of methotrexate is the rule. ${ }^{43}$ The role of steroids is controversial. In several cases, spontaneous resolution has occurred despite continued administration of methotrexate. Due to the potential for irreversible pulmonary fibrosis, and life-threatening pulmonary insufficiency, however, it seems prudent to withhold methotrexate in cases of presumed toxicity. Corticosteroids may have a role in fulminant severe cases, in an attempt to attenuate the inflammatory and fibrotic response. Unfortunately, no criteria reliably predict the 
development of methotrexate pneumonitis, and no correlation has been found with duration or total dosage of therapy. ${ }^{43}$

Other cytotoxic agents can induce similar acute and chronic pneumonitis syndromes. The roentgenographic, clinical, and histologic features are similar to those described with busulfan and methotrexate. Discontinuation of the drug implicated is recommended.

Chrysotherapy can rarely cause pulmonary injury. Chest roentgenograms may show diffuse infiltrates, of either interstitial or alveolar type, and fulminant respiratory failure can ensue. ${ }^{45}$ Lung biopsies show changes compatible with interstitial pneumonitis and fibrosis but are in no way specific. ${ }^{45}$ In the presence of associated signs of gold hypersensitivity such as exfoliative dermatitis, stomatitis, anemia, or fever, pulmonary infiltrates should be strongly considered as related to gold toxicity and the drug should be discontinued. The absence of extrapulmonary signs, however, does not exclude gold-induced lung injury. Although in some cases peripheral lymphocytes transform in response to gold, ${ }^{46}$ others have reported negative results. ${ }^{45}$ Spontaneous resolution concurrent with discontinuance of gold therapy has been reported, while others have required corticosteroid therapy. ${ }^{45}$ The exact role and proper dosage of corticosteroids is uncertain due to the paucity of well-documented cases.

\section{Intrapulmonary Hemorrhage}

Progressive dyspnea and diffuse pulmonary infiltrates due to extensive intrapulmonary hemorrhage have been reported in the absence of gross hemoptysis. ${ }^{7.47} \mathrm{Al}$ though bronchoalveolar lavage with return of bloody lavage fluid and marked increase in stainable hemosiderin within alveolar macrophages has been advocated as a diagnostic aide in occult pulmonary hemorrhage, ${ }^{47}$ positive results do not necessarily exclude infectious processes causing hemorrhage (eg, necrotizing bronchopneumonia, invasive aspergillus and Mucormycosis) and hence seem to be of limited clinical value. Occult pulmonary hemorrhage was detected in $54 \%$ of all lungs in one series of 50 consecutive leukemia patients at autopsy, but clinically significant hemorrhage was seen in only three cases, generally in association with severe thrombocytopenia. ${ }^{7}$ In the absence of severe coagulopathy or thrombocytopenia, pulmonary infiltrates in the IP due to intrapulmonary hemorrhage are rare.

\section{Noninvasive Procedures}

Routine serologies in the evaluation of pulmonary infiltrates in IPs are of little or no value. A large prospective study of diffuse pulmonary infiltrates in IPs showed essentially no discriminatory value of extensive serological testing for CMV, HSV, influenza and other viruses, Mycoplasma, Legionnaire's, P. carinii, Aspergillus, Candida, Cryptococcus, and other fungi, ${ }^{32}$ Others have con- firmed the unreliability of serologic testing for detection of $P$. carinii. ${ }^{37}$

Several investigators have affirmed the lack of specificity of the chest roentgenogram in the evaluation of IPS. ${ }^{8,26,32}$ The rapidity of change of the roentgenogram, the pattern of infiltrates, presence or absence of cavitation, pleural effusion, adenopathy, and the like may be sufficiently characteristic to render certain diagnoses either likely or improbable, but should not be relied upon to give a precise etiologic diagnosis. Although Pennington noted the inability to predict the etiology of infiltrates by the pattern on roentgenogram, he noted certain trends: bacterial pneumonia frequently presented as local consolidation ( 9 of 11 cases), and in no case displayed diffuse interstitial infiltrates. ${ }^{8}$ Fungal disease displayed variable features, including consolidation, cavities, and diffuse interstitial infiltrates. ${ }^{8}$ Similarly, the presence of cavities, pleural effusions, lymphadenopathy, or well-circumscribed nodules are sufficiently uncommon in $P$. carinii pneumonitis to render the diagnosis improbable. ${ }^{26,33,36}$

Hence, findings on chest roentgenogram may narrow the etiological possibilities to an extent. Diffuse re-ticulonodular infiltrates essentially excludes the diagnosis of bacterial pneumonia, and coverage with antibiotics alone would be wholly inadequate. Conversely, a well-circumscribed nodule or cavity is highly unlikely to represent $P$. carinii, but is compatible with mycobacterial, fungal, or bacterial causation.

\section{Invasive Techniques}

Since noninvasive techniques infrequently provide a specific etiological diagnosis, invasive techniques to obtain pulmonary parenchyma may be required. Although open lung biopsy provides the highest yield of specific diagnoses, ${ }^{26,27}$ the requirements for general anesthesia, a thoracostomy tube, and potential for serious operative complications preclude its routine use. Transbronchial lung biopsy (TBB) utilizing the fiberoptic bronchoscope, however, can be performed under local anesthesia with minimal morbidity and a specific diagnostic yield of in excess of $30-50 \% .^{8,28-31,48}$ TBB has been particularly efficacious in the diagnosis of $P$. carinii pneumonitis ${ }^{28-3.1}$ as well as fungal diseases ${ }^{8,23,28,29}$ and underlying neoplasm. ${ }^{28-30}$ Bronchial brushings in conjunction with TBB may enhance the diagnostic yield. ${ }^{8,28,31}$ Bronchial brushings and cultural techniques utilizing a sheathed catheter for accurate bacteriologic (noncontaminated) cultures may permit better evaluation of bacterial pneumonias when sputum production is minimal. ${ }^{49}$

In virtually all series, the most common TBB finding is nonspecific inflammatory changes (NSC) or fibrosis, seen in $32 \%$ to $45 \%$ of biopsies. ${ }^{25,28,31,48}$ The significance of such changes is controversial. Although Poe et al concluded that NSC are infrequently associated with specific treatable diseases, ${ }^{30}$ we found that more aggres- 
sive techniques led to specific treatable disorders in a majority of cases. ${ }^{31}$ In several such cases, the diagnosis led to initiation of new and specific therapy which resulted in recovery.

In summary, although fiberoptic bronchoscopy with TBB permits a specific etiologic diagnosis in a minority of cases, its low morbidity justifies its use as the initial invasive diagnostic procedure in IP with pulmonary infiltrates.

\section{Summary}

The approach to the IP with pulmonary infiltrates should be both thorough and immediate. Vigorous search for skin or mucous membrane lesions with appropriate aspirate, biopsy, and cultural techniques may permit early diagnosis. Sputum and blood cultures, sputum gram stain, and Papanicolau smear to demonstrate fungi, virocytes, or neoplastic cells should be performed. In patients with neurological signs, lumbar puncture to include cryptococcal antigen is indicated. When disseminated fungal disease is a consideration, urine cultures for fungi and bone marrow biopsy with cultures should be performed within 24 hours of admission. Erythromycin and TMP-SMX therapy to cover for Legionnaire's and $P$. carinii pneumonitis pending culture results seems reasonable in any IP with pulmonary infiltrates.

In patients with a clinical course compatible with bacterial illness, empirical broad-spectrum antibiotics should be begun immediately. Examination of sputum gram stain may guide therapy if a predominant organism is repeatedy visualized. In patients with a clinical diagnosis of bacterial pneumonia in whom response to antibiotics is slow, transbronchial lung biopsy (TBB) with cultures should be performed to guide therapy. If the TBB is nondiagnostic and clinical response is inadequate, open lung biopsy should be performed.

In IPs with diffuse reticulonodular infiltrates, bacterial infection is unlikely and parenteral antibiotics alone are probably inadequate. Further, due to the low yield of noninvasive techniques in such patients, TBB with special stains for fungi, $P$. carinii, and mycobacteria should be done within 24 hours of admission. If TBB is nondiagnostic, unless other tests are definitive, open lung biopsy is recommended within the next 48 to 96 hours to substantiate a diagnosis.

\section{Drug Names}

amphotericin B: Fungizone

azothiaprine: Imuran

bleomyxin: Blenoxane

busulfan: Myleran

cephalothin: Keflin

cyclophosphamide: Cytoxan

melphelan: Alkeran

6-mercaptopurine: Purinethol

trimethoprim: Bacterim, Septra

\section{References}

1. Zurier RB, Weissman G: Anti-immunologic and antiinflammatory effects of steroid therapy. Med Clin North Am 57:1295, 1973

2. Hurley DL, Balow JE, Fauci AS: Experimental disseminated candidiasis. Administration of glucocorticosteroids, susceptibility to infection and immunity. I Infect Dis 132:393, 1975

3. Sheldon WK: Experimental pulmonary Pneumocystis carinii infection in rabbits. J Exp Med 110:147, 1959

4. Anderson RI, Schafer LA, Olin DB, et al: Infectious risk factors in the immunocompromised host. Am I Med 54:453, 1973

5. Williams DM, Krick JA, Remington JS: Pulmonary infection in the compromised host. Am Rev Respir Dis 114:359, 1976

6. Sickles EA, Young VM, Greene WH, et al: Pneumonia in acute leukemia. Ann Intern Med 79:528, 1973

7. Bodey GP, Powell RD, Hersh EM, et al: Pulmonary complications of acute leukemia. Cancer 19:781, 1966

8. Pennington JE, Feldman NT: Pulmonary infiltrates and fever in patients with hematologic malignancy. Assessment of transbronchial biopsy. Am J Med 62:581, 1977

9. Valdivieso M, Gil-Extremera B, Zornoza I, et al: Gram-negative bacillary pneumonia in the compromised host. Medicine $56: 241,1977$

10. Briggs WA, Merrill JP, $\mathrm{O}^{\prime}$ Brien TF, et al: Severe pneumonia in renal transplant patients. Ann Intern Med 75:887, 1971

11. Saravolatz LD, Burch $\mathrm{KH}$, Fisher $\mathbf{E}$, et al: The compromised host and Legionnaire's disease. Ann Intern Med 90:533, 1979

12. Pasculle AW, Myerowitz RL, Rinaldo CR: New bacterial agent of pneumonia isolated from renal transplant recipients. Lancet ii: 58,1979

13. Young RC, Bennett JE, Geelhoed GW, et al: Fungemia with compromised host resistance. A Study of 70 cases. Ann Intern Med 80:605, 1974

14. Mirsky HS, Cuttner J: Fungal infection in acute leukemia. Cancer 30:348, 1972

15. Meyer RD, Young LS, Armstrong D, et al: Aspergillosis complicating neoplastic disease. Am J Med 54:6, 1973

16. Meyer RD, Rosen P, Armstrong D: Phycomycosis complicating leukemia and Iymphoma. Ann Intern Med 77:871, 1972

17. Cohen MS, Brook CJ, Naylor B, et al: Pulmonary phycomycetoma in a patient with diabetes mellitus. Am Rev Respir Dis 116:519, 1977

18. Davies SF, Khan M, Sarosi GA: Disseminated histoplasmosis in immunologically suppressed patients. Am J Med 64:94, 1978

19. Davies SF, McKenna RW, Sarosi GA: Trephine biopsy of the bone marrow in disseminated histoplasmosis. Am I Med 67:617. 1979

20. Rifkind D, Marchioro TL, Schneck SA, et al: Systemic fungal infections complicating renal transplantation and immunosuppressive therapy. Clinical, microbiologic, neurologic and pathologic features. Am J Med 43:28, 1967

21. Kaplan $\mathrm{MH}$, Rosen PP, Armstrong D: Cryptococcosis in a cancer hospital. Clinical and pathological correlates in forty-six patients. Cancer 39:2265, 1977

22. Fisher BD, Armstrong D: Cryptococcal interstitial pneumonia. Value of antigen determination. N Engl I Med 297:1440, 1977

23. Sanders JS, Sarosi CA, Nollet DJ, et al: Exfoliative cytology in the rapid diagnosis of pulmonary blastomycosis. Chest 72:193, 1977

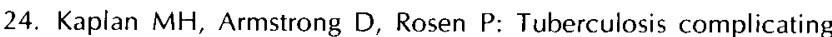
neoplastic disease. A review of 201 cases. Cancer 33:850, 1974

25. Feld R, Bodey GP, Groschel D: Mycobacteriosis in patients with malignant disease. Arch Intern Med 136:67, 1976

26. Greenman RL, Goodall PT, King D: Lung biopsy in immunocompromised hosts. Am J Med 59:488, 1975

27. Rossiter SJ, Miller DC, Churg AM, et al: Open lung biopsy in the immunosuppressed patient. Is it really beneficial? J Thorac Cardiovasc Surg 77:338, 1979

28. Cunningham JH, Zavala DC, Corry RJ, et al: Trephine air drill, bronchial brush, and fiberoptic transbronchial lung biopsies in immunosuppressed patients. Am Rev Respir Dis 115:213, 1977

29. Matthay RA, Farmer WC, Odero D: Diagnostic fiberoptic bronchoscopy in the immunocompromised host with pulmonary infiltrates. Thorax 32:539, 1977 
30. Poe RH, Utell MJ, Israel RH, et al: Sensitivity and specificity of the nonspecific transbronchial lung biopsy. Am Rev Respir Dis 119:25, 1979

31. Nishio JN, Lynch JP: Fiberoptic bronchoscopy in the immunosuppressed host: the significance of a 'nonspecific' transbronchial biopsy. Am Rev Respir Dis 121:307, 1980

32. Singer C, Armstrong D, Rosen PP, et al: Diffuse pulmonary infiltrates in immunosuppressed patients. Prospective study of 80 cases. Am J Med 66:110, 1979

33. Rosen P, Armstrong D, Ramos C: Pneumocystis carinii pneumonia. A clinicopathologic study of twenty patients with neoplastic diseases. Am J Med 53:428, 1972

34. Goodell B, Jacobs JB, Powell RD, et al: Pneumocystis carinii: the spectrum of diffuse interstitial pneumonia in patients with neoplastic diseases. Ann Intern Med 72:337, 1970

35. Walzer PD, Perl DP, Krogstad D], et al: Pneumocystsis carinii pneumonia in the United States: epidemiologic, diagnostic and clinical features. Ann Intern Med 80:83, 1974

36. Doppman JL, Geelhoed GW, DeVita VT: Atypical radiographic features in pneumocystis carinii pneumonia. Radiology 114:39, 1975

37. Meyers JD, Pifer LL, Sale GE, et al: The value of pneumocystis carinii antibody and antigen detection for diagnosis of pneumocystis pneumonia after marrow transplantation. Am Rev Respir Dis 120:1283, 1979

38. Hughes WT: Pneumocystis carinii pneumonia. N Engl J Med 297:1381, 1977
39. Triebwasser $\mathrm{JH}$, Harris $\mathrm{RE}$, Bryant $E R$, et al: Varicella pneumonia in adults. Medicine 46:409, 1967

40. Craighead IE: Pulmonary cytomegalovirus infection in the adult. Am J Pathol 63:487, 1971

41. Dudding BA, Wagner SC, Zeller JA, et al: Fatal pneumonia as.sociated with adenovirus type 7 in three military trainees. $N$ Engl J Med 286:1289, 1972

42. Gross NJ: Pulmonary effects of radiation therapy. Ann Intern Med $86: 81,1977$

43. Sostman HD, Matthay RA, Putman CE, et al: Methotrexateinduced pneumonitis. Medicine 55:371, 1976

44. Burns WA, McFarland W, Mathews MJ: Busulfan-induced pulmonary disease: report of a case and review of the literature. Am Rev Respir Dis 101:408, 1970

45. Winterbauer RH, Wilske KR, Wheelis RF: Diffuse pulmonary injury associated with gold treatment. N Engl J Med 294:919, 1976

46. Geddes D, Brostoff J: Pulmonary reaction to chrysotherapy, $\mathrm{N}$ Engl J Med 295:506, 1976

47. Fingley TN, Aronow A, Cosentino AM, et al: Occult pulmonary hemorrhage in anticoagulated patients. Am Rev Respir Dis $112: 23,1975$

48. Feldman NT, Pennington JE, Ehrie MG: Transbronchial lung biopsy in the compromised host. JAMA 238:1377, 1977

49. Wimberley N, Faling LJ, Bartlett JG: A fiberoptic bronchoscopy technique to obtain uncontaminated lower airway secretions for bacterial cultures. Am Rev Respir Dis 119:337, 1979
STATEMENT OF OWNERSHIP, MANAGEMENT AND CIRCULATION (Act of August 12, 1970: Section 3685. Title 39. United States Code)

Date of Filing-September 25, 1980

Title of Publication-International Journal of Dermatology

Frequency of Issue-Ten Issues Per Year (Monthly except Feb, Aug)

Annual Subscriptions: $\$ 33.00$ U.S. and Possessions; $\$ 20.00$ resident rate (U.S. only); $\$ 38.00$ all other countries except Japan. Single copies $\$ 5.00$.

Location of Known Office of Publication-East Washington Square, Philadelphia, PA 19105

Location of the Headquarters or General Business Offices of the Publishers-East Washington Square, Philadelphia, PA 19105

Publisher-J. B. Lippincott Company, East Washington Square, Philadelphia, PA 19105

Editor-Raul Fleischmajer, M.D., One Gustave Levy Place, New York, NY 10029

Managing Editor-Katherine Subramanian, East Washington Square, Philadelphia, PA 19105

Owner-International Society of Tropical Dermatology, Three E. 69th St., New York, NY 10021

Known Bondholders, Mortgagees and other security holders owning or holding 1 percent or more of total amounts of bonds, mortgages or other securities-None.

Nonprofit Organizations Authorized to Mail at Special Rates-The purposes, functions, and non-profit status of this organization and the exempt status for Federal income tax purposes have not changed during preceding 12 months.
A. Total no. copies printed (net press run)

B. Paid circulation

1. Sales through dealers and carriers, street vendors and counter sales

2. Mail subscriptions

C. Total paid circulation

D. Free distribution by mail,

carrier or other means. Samples, complimentary, and other free copies

$\mathrm{E}$. Total distribution (sum of $\mathrm{C}$ and

D)

F. Copies not distributed

1. Office use, leftover, unaccounted, spoiled after printing

2. Returns from news agents

G. Total (sum of E \& F-should equal net press run shown in A)

Average No.
Copies Each
Issue during
Preceding
12 Months

3,605

451

2,349

2,808

99

2,907

698

Actual No.

of Copies of

Single Issues

Published

Nearest to

Filing Date

3,600

I certify that the statements made by me above are correct and complete. 
This document is a scanned copy of a printed document. No warranty is given about the accuracy of the copy. Users should refer to the original published version of the material. 